\title{
WORKFORCE Intensive care medicine in smaller hospitals: here to stay
}

\author{
Author: Christopher M Thorpe ${ }^{\mathrm{A}}$
}

\begin{abstract}
Intensive care medicine is a relatively new specialty. In developing standards of care, it became apparent that some aspects were not achievable by smaller units. Within the intensive care community, there has been a gradual acceptance that smaller hospitals cannot necessarily implement structures that are used in large hospitals, and that outcomes can be comparable with larger units despite this. The Faculty of Intensive Care Medicine set up a Smaller and Specialist Units Advisory Group to explore this area, and this article initially explains the background and work of the faculty to support and sustain these units. We then move on to look at critical care in the context of the recent emergence of wider work on remote and rural healthcare. Finally, we explore our future horizons and look in detail at the areas where further developments will transform the care of critically ill patients within the smaller hospitals of the next 20 years.
\end{abstract}

KEYWORDS: Intensive care medicine, smaller hospitals, critical care

\section{Introduction}

Smaller hospitals have difficulties in delivering healthcare in the same format as larger hospitals. Research over the last few years, led by the Nuffield Trust and The King's Fund, has emphasised that, although the structure of delivery may vary, good care can occur in all sizes of hospital and volume effects on outcomes are limited to just a few specialties. ${ }^{1,2}$ This has led to an increased interest in preserving and developing care in smaller hospitals, and in particular those that are remote and rural. This approach is mirrored by changes in the intensive care community, and this article explains the background behind the work done to date to support these units, and then looks at potential future developments.

\section{Background}

Intensive care medicine (ICM) has a critical role to play in providing high quality care to patients admitted to an acute

Author: ${ }^{A}$ consultant in anaesthetics and intensive care medicine, Ysbyty Gwynedd, Bangor, UK and chair of the Smaller and Specialist Units Advisory Group, The Faculty of Intensive Care Medicine, London, UK hospital. In the past, ICM has been joined at the hip with anaesthesia - up until 9 years ago there was no route to train as an intensivist alone, and the vast majority of consultants working in the field were trained in anaesthesia as their primary specialty. The Faculty of ICM (FICM) was set up in 2010, following years of work by the Intensive Care Society and the Intercollegiate Board for Intensive Care Medicine, and now develops and maintains the training for intensivists across the UK. ${ }^{3}$ There has been an increase, year on year, of trainees appointed to the training scheme. Many still combine this training with another specialty, commonly anaesthesia, medicine or emergency medicine, but increasing numbers are training in ICM alone. ${ }^{4}$

This development has resulted in a clear dividing line between trained intensivists, with a specialist qualification in ICM and other doctors who have ICM training as part of another specialty programme. The concept that only trained intensivists would provide care for critically ill patients has driven the UK ICM community to develop systems, standards and training programmes to further this aim. ${ }^{5}$ Many of these have been successful, and clear national standards for delivery of critical care have helped shine a light on the specialty, and enabled local services to obtain extra funding.

In keeping with guideline development in other specialties, the impetus has come from the national bodies that have navigated their way through political and financial barriers to effect the change in care delivery. Also in common with other specialties, the national bodies are heavily populated with doctors based in tertiary centres. As a result, two aspects have emerged.

Firstly, on a broader canvas a school of thought emerged that emphasised that better care was being delivered by big centres, and also the economy of scale that delivered financial savings. ${ }^{6,7}$ This campaign was so effective that it has been embedded into the collective consciousness of both clinicians and political bodies. For a long period of time, this went unchallenged and the upshot from this was that smaller hospitals became increasingly concerned about their viability. Crucially, financial and political backup for this strategy was half-hearted at best and smaller hospitals were faced with the unenviable position of continuing to care for acutely ill patients while the zeitgeist continued to emphasise the tertiary centre as the future of hospital medicine. ${ }^{8}$ Secondly, the effect on critical care was that standards produced were heavily weighted towards the larger, more resourced tertiary centres leading to pressure on both clinical teams and management to alter the service. This led to increased finance going into the specialty, and some units benefited from improved resources. Some 
units, however, were unable to benefit for two reasons: firstly the size of the unit meant that clinical workload was not sufficient to support staffing along the lines put forward in the standards and secondly a deficit in the number of staff available nationally, both trained intensivists and resident doctor numbers, meant that there were nowhere near enough personnel to support the expansion proposed.

As a result of changes introduced, the system was purposefully stressed. While this empowered some hospitals to gain resource, it became clear that some smaller units could not feasibly match standards introduced. Importantly, many of these smaller units in the UK defied the political assumption that bigger is better and demonstrated outcomes that matched larger units, even with adjusted staffing structures. ${ }^{9}$

A difference of opinion led to interesting discussions among the ICM community. In a nutshell, some believed smaller units should close and become stabilisation and transfer centres; others believed that onsite critical care expertise was an essential component of a safe hospital. The FICM set up a Smaller and Specialist Units Advisory Group (SSUAG) to explore this area and to provide evidence-based guidance.

The SSUAG comprises representatives from smaller hospitals in all four countries of the UK. It reports directly to the board of the FICM, and has a remit of ensuring that smaller hospitals are considered when new policies or documents are produced. It is also charged with investigating difficulties and developing solutions for service delivery in these hospitals.

The difficulties faced by smaller units in the UK are replicated in other countries. For example, the USA has a lack of trained intensivists and the effect of this shortage is felt in smaller rural hospitals particularly. Some hospitals have no trained intensivists and in others the model varies between closed and open units. ${ }^{10,11}$ Patients in closed units are primarily looked after by doctors based within the unit while patients in open units are primarily looked after by the admitting specialty doctor. Closed units are held to be more effective and evidence, although conflicted, favours this model. Given the inability to fully staff units with trained intensivists, a variety of models emerged where the input from this limited resource was combined with help from other sources. In particular, night-time work on a frequent rota is both unattractive and difficult to sustain in a busy acute specialty and recent evidence suggests that as long as trained specialists cover daytime work, there is no increase in mortality if night-time work does not have ICM specialists as the primary responder. ${ }^{12,13}$ Germany accredits intensivists through a period on top of their anaesthetic training, and does not have a separate college. They have a large number of hospitals, with 1,197 anaesthetic departments in the country. An evaluation of 993 hospitals showed that more than $90 \%$ had their own intensive care unit. ${ }^{14}$ Even given the larger population of Germany, around 83 million, this number of intensive care units dwarfs the 210 units in the UK. Smaller units in Germany have similar problems in staffing critical care and tend to have anaesthetists supporting the rota at night.

Telemedicine is one area that has developed within the USA to help supply ICM input to smaller hospitals, but this is not without its drawbacks. These systems involve a fully staffed hub linked in real time to a variety of units that do not have trained intensivists on site. The method of fully integrated telemedicine service has been explored by Farmer, and the costs are high, both financial and staffing. ${ }^{15}$ There still needs to be an on-site clinician able to deliver the care, even though decision making can be shared and supported remotely.
A more pragmatic solution could be explored which involves networked support, possibly supported by remote access to monitors and notes via a clinical informatics system. This could potentially give most of a full telemedicine service at a fraction of the cost. This arrangement makes use of a notional division between the tactical skills needed to deliver task-based care at the bedside, and the more strategic decision making that can be more easily delivered by trained intensivists. ${ }^{16}$ This division runs through all of hospital-based care in the UK. It is accepted in most specialties that the consultant supervises but does not deliver all the care for all patients. This is particularly true at night, where strategic decisions on service and patient care are much less common, and consultants would generally come in either for taskbased care that is beyond resident staff or for situations where demand has outstripped supply.

\section{Current state of play}

Following discussions and negotiations within the specialty, the latest version of the bible for delivery of critical care services now contains a chapter on remote and rural units. The first edition of Guidelines for the provision of intensive care services, published in 2015 , had been a big endeavour for a newly formed specialty. Running to 200 pages, it covered everything from the physical structure to the number of doctors needed. ${ }^{5}$ Although extremely helpful to the specialty as a whole, a small minority of chapters contained standards that proved impossible to meet for some smaller hospitals. This caused anguish and discontent, and it was imperative that the next version addressed the problem. ${ }^{17} \mathrm{~A}$ targeted chapter on remote and rural units was incorporated. ${ }^{18}$ This chapter provided 10 standards and four recommendations (Box 1). This has legitimised the service structure in place in many smaller units, and the standards are aimed at a level where realistic improvements could be made.

Guidance for training has recently been updated by FICM, with a specific paragraph on attachments to smaller units, hopefully leading the way to increased exposure and improved recruitment.

\section{Where next?}

How can we develop critical care services? It is easy to blame workforce shortages, and a lack of trained intensivists, for an inability to staff a rota entirely with intensivists $24 / 7$. While it is true that there is not the workforce available, it is also true that we need to work smarter and look at practical ways to deliver safe care. $^{19}$

\section{Staffing}

A critical concept is that having a trained intensivist guiding care in your hospital is a great benefit, and can work both as a quality assurance role to ensure that care delivered to deteriorating patients is appropriate and in keeping with accepted practice; and also as a catalyst to improve recruitment and retention. The responsibility of supervising the service weighs heavily on a single consultant, and there should be a cohort of intensivists to share the burden. Smaller hospitals will need to gradually increase the number of trained intensivists. Recruitment is best achieved by fitting into a service that is already functional and offers a good work-life balance. These systems will have been established with a broad range of staff, and retention of these staff to complement 
Box 1. Smaller remote and rural critical care units. Reproduced with permission from The Faculty of Intensive Care Medicine, Intensive Care Society. Guidelines for the provision of intensive care services. FICM, 2019.

\section{Standards}

Network support must be in place to ensure smaller, remote and rural critical units meet these standards and recommendations.

The critical care service must be led by consultants trained in ICM.

There must be access to appropriate advice from a consultant in ICM at all times.

Dedicated daytime critical care must be provided by a consultant trained in ICM with no other commitments.

There must be a doctor or ACCP with advanced airway skills resident within the hospital $24 / 7$.

There must be a 24/7 dedicated resident on the critical care unit

There must be structured handover between daytime and nighttime staff supported by standardised policies for practice.

Appropriate CPD must be supported by the employer and undertaken by all professionals who deliver intensive care.

Regional transport arrangements (road and air) must be put in place to allow timely, safe transfer of patients with an appropriate level of monitoring, staffing and skills.

All critical care units, including level 2 units, must enter data into national databases such as ICNARC or SICSAG.

\section{Recommendations}

Network support should be explicit, resourced and supported by all the healthcare organisations, boards, networks and regions involved, and recognised in job planning.

Units should consider the development of telemedicine techniques for clinical decision making and educational support, in conjunction with their regional network.

Remote critical care units should implement appropriate joint clinical governance procedures with both networked units and transfer services to include case-based review, critical incident analysis and joint educational sessions.

Where an intensive care pharmacist or healthcare professional, such as a physiotherapist or dietitian, cannot be effectively delivered locally in a small unit, advice should be accessible from specialist colleagues through network support. Appropriate training bodies should devise and support remote and rural training posts in critical care.

$\mathrm{ACCP}=$ acute critical care practitioner; $\mathrm{CPD}=$ continuing professional development; ICM = intensive care medicine; ICNARC = Intensive Care National Audit \& Research Centre; SICSAG = Scottish Intensive Care Society Audit Group.

the incoming intensivists will sustain a workable system. Returning to the concept of tactical (task-based) care, the majority of nighttime work will therefore be carried out by a pool of skilled staff well trained in dealing with a sick patient. At a consultant level, this will be a combination of intensivists and anaesthetists, with some physicians and emergency medicine physicians as part of the mix.
Increasing the number of trained intensivists into this system is far easier than trying to recruit into a frequent unsupported rota in a remote hospital. The quality of work-life balance is crucial in recruitment to smaller hospitals, and the hardship of the on-call rota is an important component.

At a resident level, there will be an increased number of acute critical care practitioners (ACCPs). ACCPs usually have a nursing background, frequently from critical care, and are trained to take on a junior doctor role. So far, they have proved excellent additions to the team and, although at the early stages of development, have shown independent practice in a wide range of critical care skills, they will be supported within the hospital by resident anaesthetic staff. $^{20}$

It will be accepted that an essential part of delivering care in smaller hospitals is the blurring of silo roles. At a consultant level, an anaesthetist with a strong critical care skill set may work with a physician to provide a package of care to a patient that rivals a single intensivist. Similarly, at a resident level, the ACCP may work alongside a trainee anaesthetist to provide a strong tactical team on site.

\section{Outreach}

Care of the deteriorating patient on the ward will involve an adjusted outreach team. Currently the model runs from the ICU, and care is escalated along these lines. The outreach system works well when there is enough 'dose' of intensive care expertise to cope with all the referred ill patients in the hospital. Where it falls down is the tendency for ward teams to take a step back and allow the critical care team to deal with all problems, including those that do not warrant critical care. This can lead to further deskilling of medical consultants, and an increased burden on the critical care team. In smaller hospitals, this model will alter so that outreach falls under the medical team as an acute intervention team (AIT). Consultants and their teams on the ward therefore remain engaged with their ill patients. The AIT system will also link with ICM and the system will operate as a whole, and act as a buffer to silo working, preserving a wider range of critical care skills within the hospital.

\section{Competencies}

A risk for smaller hospitals is the gradual eroding of competencies as individual diseases or age groups may be seen infrequently. ${ }^{18}$ Paediatrics is a particular case in point. Jobs in smaller hospitals will therefore be linked with larger units so to preserve and develop competencies. Where a small hospital is within a reasonable distance to larger hospitals, a shared job is a practical solution. For more remote units, attachments will be part of the local contract, in addition to the usual study and professional leave. Networked support will incorporate joint training programmes, linked by teleconference where necessary. Links to larger units will also be extended to nursing and other staff maintaining a wider group benefiting from this process.

\section{Transfers}

Transfers of critically ill patients denude smaller hospitals of vital staff. A rapid upsurge in prehospital care will overspill into transfer of these patients and there will be separate professional teams who will retrieve patients throughout the UK. They will be funded for both road and air travel, and so patients with particular needs, 
for example liver failure or multiple trauma, will be transferred directly to the appropriate facility. An increased capability for night flying will enable the service to run $24 / 7 .^{21}$

\section{Networks}

Smaller units will be embedded in functional clinical networks. Shared quality assurance processes and annual reporting will closely link units, and consultant familiarity will be cemented by regular attachments and tele-linkage. Clinical information systems will allow the sharing of data and live monitoring information to improve remote collaboration between units. Established networks in Holland show the benefits and sustainability of well-run critical care networks in supporting smaller units. ${ }^{22}$

\section{Complexity}

Further research will uncover the link between tactical skill set and complexity. Smaller ICUs benefit hugely from the limited number of patients that need to be cared for at any one time. Units with eight patients are much easier to manage for a single consultant and resident, with recent evidence suggesting this number is around the optimum. ${ }^{23}$ The handover is relatively straightforward and it is easier to retain all patients in your head, and to remember details. This is an enormous benefit to the on-call team, and is probably one of the reasons why non-intensivists can safely manage night-time work in smaller units.

\section{Training}

There will be an increase in the number of trainees rotating through smaller units. Doctors generally like consultant jobs in places where they have trained, so increasing the trainee attachments will result in increased recruitment. In addition, training will be altered by the General Medical Council so that fellowship posts can be accredited retrospectively assuming they meet educational standards. This will lead to a new cohort of trainees, who like ICM but do not wish to base their life in urban centres. They will move through training while preserving their work-life balance locally for a large proportion of their progression, working in larger urban hospitals for targeted discrete attachments. This new cohort will have a different approach and new ways of working, for example splitting their working life between general practice and intensive care medicine.

\section{Retirement}

Finally, on a wider scale the government will accept that hardpressed specialties are akin to the police and armed forces. The ability to deliver safe care 24 hours a day in an acute setting affects both personal health and patient safety as the healthcare professional gets older. ${ }^{24}$ The pension will be able to be taken without penalty at age 60 . This will lead to the resurgence of intensive care medicine as an achievable career and both recruitment and retention will dramatically improve, leading to an exemplar first-class service throughout all sizes of hospital.

\section{Conclusion}

There is currently still a problem with supply, both in terms of personnel and finance, which hamper plans to develop critical care in smaller hospitals. The service therefore needs to adapt and the first steps in this process are in place. The acceptance from the intensive care community that one size does not fit all allows smaller units to develop the platform for sustainable, high-quality care. Closer links are already being forged between larger and smaller units, and we can expect smaller hospitals to continue to be served by onsite critical care for many years to come.

\section{References}

1 Imison C, Sonola L, Honeyman M, Ross S. The reconfiguration of clinical services: what is the evidence? London: The Kings Fund, 2014.

2 Watson R, Crump H, Imison C, Currie C, Gaskins M. Emergency general surgery: challenges and opportunities: Research report. London: Nuffield Trust, 2016.

3 Bion J. History of the FICM. The Faculty of Intensive Care Medicine. www.ficm.ac.uk/faculty-membership/history-ficm.

4 The Faculty of Intensive Care Medicine. Workforce data bank for adult critical care. FICM, 2018. www.ficm.ac.uk/sites/default/files/ workforce_data_bank_2018_updated_for_website.pdf

5 The Faculty of Intensive Care Medicine, Intensive Care Society. Guidelines for the provision of intensive care services. FICM, 2015.

6 Healthcare for London. A framework for action. NHS, 2007.

7 Soljak M. Volumes of procedures and outcome of treatment. BMJ 2002;325:787-8.

8 Suntharalingam G, Handy J, Walsh A. Regionalisation of critical care: Can we sustain an intensive care unit in every hospital? Anaesthesia 2014:69:1069-73.

9 Wilcox ME, Harrison DA, Short A, Jonas M, Rowan KM. Comparing mortality among adult, general intensive care units in England with varying intensivist cover patterns: a retrospective cohort study. Crit Care 2014;18:491.

10 Checkley W, Martin GS, Brown SM et al. Structure, process, and annual ICU mortality across 69 centers: United States Critical Illness and Injury Trials Group Critical Illness Outcomes Study. Crit Care Med 2014:42:344-56.

11 Yoo EJ, Edwards JD, Dean ML, Dudley RA. Multidisciplinary critical care and intensivist staffing: results of a statewide survey and association with mortality. J Intensive Care Med 2014:31:325-32.

12 Kerlin MP, Small DS, Cooney E et al. A randomized trial of nighttime physician staffing in an intensive care unit. N Engl J Med 2013;368:2201-9.

13 Wallace DJ, Angus DC, Barnato AE, Kramer AA, Kahn JM. Night time intensivist staffing and mortality among critically ill patients. N Engl J Med 2012;366:2093-101.

14 Deutsche Gesellschaft für Anästhesiologie und Intensivmedizin. About us: The German Society of Anaesthesiology and Intensive Care Medicine. Nürnberg: DGAI. www.dgai.de/die-dgai/aufgabenund-ziele/about-us.html

15 Farmer JC. Important administrative aspects of critical care telemedicine programs. Crit Care Clin 2019;35:407-14.

16 Fackler JC, Watts C, Grome A et al. Critical care physician cognitive task analysis: an exploratory study. Crit Care 2009;13:R33.

17 The Faculty of Intensive Care Medicine, Intensive Care Society. Guidelines for the provision of intensive care services. FICM, 2019. www.ficm.ac.uk/sites/default/files/gpics-v2.pdf.

18 Thorpe CM, Barr C. Smaller remote and rural critical care units. In: The Faculty of Intensive Care Medicine, Intensive Care Society. Guidelines for the provision of intensive care services. FICM, 2019:71-4.

19 Thorpe C, Vaughan L. Specialist not tertiary: Providing intensive care medicine in a district general hospital. J Intensive Care Soc 2018;19:92-3. 
20 Denton G, Green L, Palmer M et al. The provision of central venous access, transfer of critically ill patients and advanced airway management: Are advanced critical care practitioners safe and effective? J Intensive Care Soc 2019;20:248-54.

21 Nutbeam T, Leaman A, Oakley P. Transporting major trauma patients from the margins of a UK trauma system. Emerg Med J 2012;29:182-3.

22 Kluge GH, Brinkman S, van Berkel $\mathrm{G}$ et al. The association between ICU level of care and mortality in the Netherlands. Intensive Care Med 2015;41:304-11.

23 Gershengorn HB, Harrison DA, Garland A et al. Association of intensive care unit patient-to-intensivist ratios with hospital mortality. JAMA Intern Med 2017;177:388-96.
24 Jones $\mathrm{A}$, Walker ], Thorpe CM. Increasing the retirement age in intensive care medicine: Perception of the effect on patient safety and personal health. J Intensive Care Soc 2014:15:57-60.

Address for correspondence: Dr Christopher M Thorpe, Department of Anaesthetics, Ysbyty Gwynedd,

Bangor LL57 2PW, UK.

Email: christhorpe@me.com

\section{Royal College of Physicians}

\section{Guidance on safe medical staffing}

The RCP's Guidance on safe medical staffing working party report aims to help those planning and organising core hospital medical services to answer the question: 'How many doctors or their alternatives, with what capabilities, do we need to provide safe, timely and effective care for patients with medical problems?'.

\section{Download the report at: www.rcplondon.ac.uk/safe-medical-staffing}
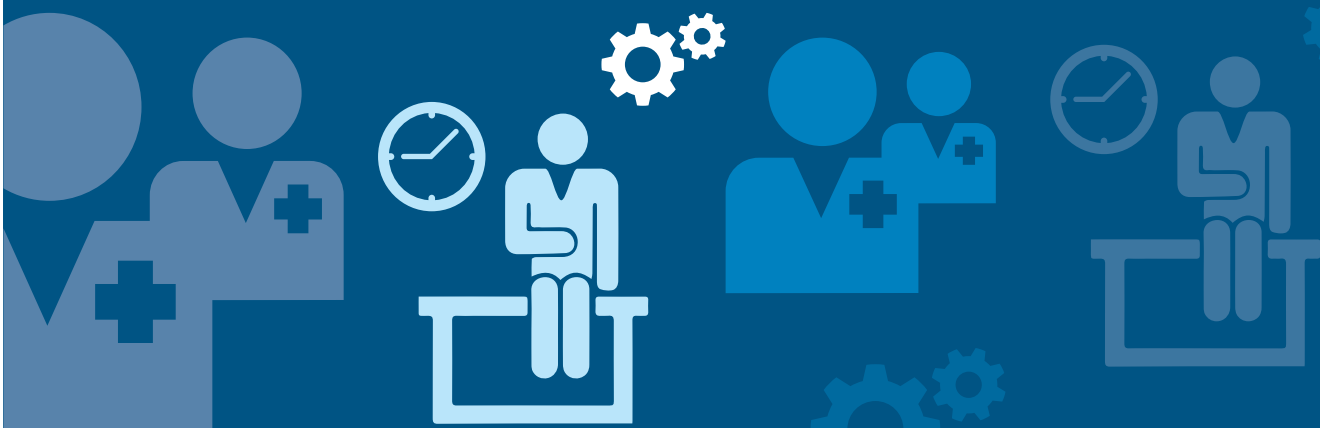